\title{
Reversal of diet-induced hepatic steatosis and hepatic insulin resistance by antisense oligonucleotide inhibitors of acetyl-CoA carboxylases 1 and 2
}

\author{
David B. Savage, ${ }^{1}$ Cheol Soo Choi, ${ }^{1}$ Varman T. Samuel,1 Zhen-Xiang Liu, ${ }^{1}$ \\ Dongyan Zhang, ${ }^{1}$ Amy Wang, ${ }^{1}$ Xian-Man Zhang, ${ }^{1}$ Gary W. Cline, ${ }^{1}$ Xing Xian Yu, ${ }^{2}$ \\ John G. Geisler,, Sanjay Bhanot, ${ }^{2}$ Brett P. Monia, ${ }^{2}$ and Gerald I. Shulman 1,3,4
}

${ }^{1}$ Department of Internal Medicine, Yale University School of Medicine, New Haven, Connecticut, USA. ${ }^{2}$ sis Pharmaceuticals, Carlsbad, California, USA. ${ }^{3}$ Department of Cellular and Molecular Physiology and ${ }^{4}$ Howard Hughes Medical Institute, Yale University School of Medicine, New Haven, Connecticut, USA

\begin{abstract}
Hepatic steatosis is a core feature of the metabolic syndrome and type 2 diabetes and leads to hepatic insulin resistance. Malonyl-CoA, generated by acetyl-CoA carboxylases 1 and 2 (Acc1 and Acc2), is a key regulator of both mitochondrial fatty acid oxidation and fat synthesis. We used a diet-induced rat model of nonalcoholic fatty liver disease (NAFLD) and hepatic insulin resistance to explore the impact of suppressing Acc1, Acc2, or both Acc1 and Acc2 on hepatic lipid levels and insulin sensitivity. While suppression of Acc1 or Acc2 expression with antisense oligonucleotides (ASOs) increased fat oxidation in rat hepatocytes, suppression of both enzymes with a single ASO was significantly more effective in promoting fat oxidation. Suppression of Acc1 also inhibited lipogenesis whereas Acc2 reduction had no effect on lipogenesis. In rats with NAFLD, suppression of both enzymes with a single ASO was required to significantly reduce hepatic malonyl-CoA levels in vivo, lower hepatic lipids (long-chain acyl-CoAs, diacylglycerol, and triglycerides), and improve hepatic insulin sensitivity. Plasma ketones were significantly elevated compared with controls in the fed state but not in the fasting state, indicating that lowering Acc1 and -2 expression increases hepatic fat oxidation specifically in the fed state. These studies suggest that pharmacological inhibition of Acc1 and -2 may be a novel approach in the treatment of NAFLD and hepatic insulin resistance.
\end{abstract}

\section{Introduction}

Nonalcoholic fatty liver disease (NAFLD) is the most frequent cause of abnormal liver function tests in the US, with an estimated prevalence of $14-20 \%(1,2)$. It is caused by triglyceride (TG) accumulation within the liver and can either be a benign self-limiting state or a condition associated with steatohepatitis, which may progress to end-stage liver disease requiring liver transplantation. The most common disorder associated with NAFLD is insulin resistance, an association which holds true in both lean and obese subjects $(3,4)$. Insulin resistance is in turn a key element in the pathogenesis of type 2 diabetes and the metabolic syndrome. Overexpressing lipoprotein lipase (LPL) in the liver in mice results in fatty liver and liver-specific insulin resistance (5). Short-term high-fat feeding in rats leads to hepatic steatosis, impaired insulin signaling, and hepatic insulin resistance, and reducing lipid accumulation with a mitochondrial uncoupling agent (2,4-dinitrophenol) improves hepatic insulin sensitivity (6). We have also shown that moderate weight loss in humans with type 2 diabetes lowers liver TGs and specifically improves hepatic insulin sensitivity (7).

Nonstandard abbreviations used: Acc, acetyl CoA carboxylase; ASO, antisense oligonucleotide; ASOctrl, ASO control; CPT1, carnitine palmitoyltransferase 1; DAG, diacylglycerol; Foxo1, forkhead transcription factor FoxO1; G6P, glucose-6 phosphatase; HGP, hepatic glucose production; LCCoA, long-chain acyl-CoA; NAFLD, nonalcoholic fatty liver disease; PEPCK, phosphoenolpyruvate carboxykinase; TG, triglyceride. Conflict of interest: Xing Xian Yu, John G. Geisler, Sanjay Bhanot, and Brett P. Monia own stock and/or hold stock options in Isis Pharmaceuticals. Citation for this article: J. Clin. Invest. doi:10.1172/JCI27300.
Together, these data strongly suggest that lipid accumulation in the liver is directly responsible for the subsequent development of hepatic insulin resistance and increased endogenous glucose production, key elements in the pathogenesis of type 2 diabetes. They also suggest that reducing liver lipid accumulation may be an attractive therapeutic strategy for NAFLD and type 2 diabetes.

Acetyl-CoA carboxylase (Acc) catalyzes the synthesis of malonylCoA, which is both an intermediate in fatty acid synthesis and an allosteric inhibitor of carnitine palmitoyltransferase 1 (CPT1) $(8,9)$. CPT1 regulates the transfer of long-chain acyl-CoAs (LCCoAs) from the cytosol into the mitochondria, where they are oxidized. Malonyl-CoA is therefore a key physiological regulator of both fatty acid synthesis (10) and oxidation (11). There are 2 isoforms of Acc in rodents and humans; Acc1 is highly expressed in liver and adipose tissue whereas Acc2 is predominantly expressed in heart and skeletal muscle and, to a lesser extent, in the liver (12). Despite being encoded by separate genes, ACC1 and ACC2 exhibit considerable sequence identity ( $\sim 85 \%$ excluding the $\mathrm{N}$ terminal extension of ACC2) and have the same domain structure responsible for enzyme activity. The primary structural difference between the 2 is an extra $\mathrm{N}$ terminal hydrophobic domain in Acc2 that appears to facilitate Acc2 localization to the mitochondrial membrane (13), where it is believed to regulate local malonyl-CoA levels, CPT1 activity, and fat oxidation. ACC2-null mice $\left(A C C 2^{-/}\right)$are leaner than wild-type controls due to increased fat oxidation in heart and skeletal muscle (12). Interestingly, hepatic fat oxidation was also increased and hepatic steatosis decreased 


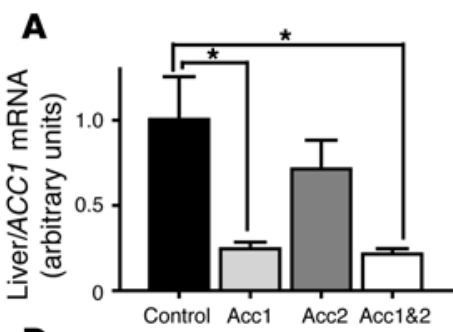

D

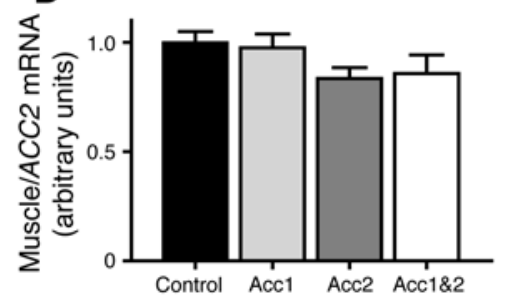

B

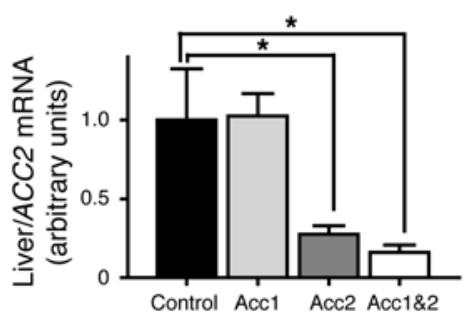

$\mathbf{E}$

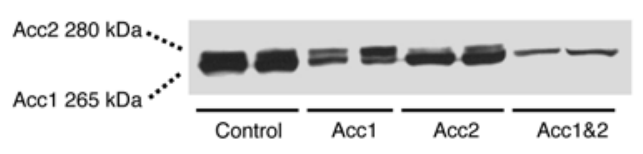

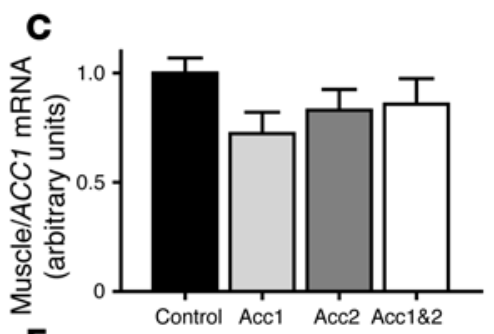

$\mathbf{F}$

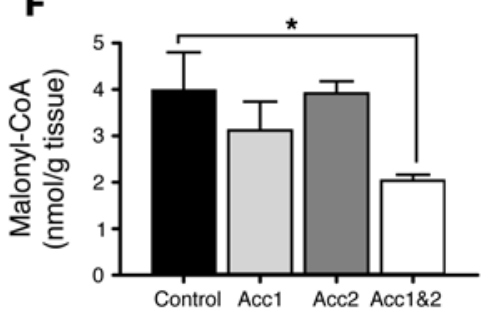

Figure 1

Acc ASOs decrease ACC expression and lower malonyl-CoA levels in rat liver. After 4 weeks of ASO treatment, total RNA was isolated from liver $(\mathbf{A}$ and $\mathbf{B})$ and muscle (C and $\mathbf{D})$, and gene expression (ACC1 mRNA [A and $\mathbf{C}$ ] and ACC2 mRNA [B and D]) was then assessed by real-time RT-PCR. Hepatic Acc1 (265 kDA) and Acc2 $(280 \mathrm{kDa})$ protein levels were assessed by Western blot analysis (E). Malonyl-CoA concentration was measured in liver homogenates from rats in the fed state $(\mathbf{F})$. Data are expressed as mean values \pm SEM of 4-6 rats per treatment group. Acc1\&2, Acc1 and Acc2. ${ }^{*} P<0.05$ versus ASOctrl treatment group.

in $A C C 2^{-/-}$mice despite normal malonyl-CoA levels; the authors postulated that this might have been a consequence of localized lowering of malonyl-CoA levels in the vicinity of mitochondria and CPT1, a change not discernible in whole-cell homogenates (12). Acc1 is localized in the cytosol, where it is thought to regulate lipogenesis in the liver and adipose tissue. ACC1-null mice are embryonic lethal (14).

Here we sought to address 2 main questions: (a) What are the respective roles of Acc1 and Acc2 in regulating fat synthesis and fat oxidation in the liver, the principal organ in which both fat synthesis and fat oxidation occur to a significant degree? and (b) What are the metabolic consequences of pharmacological manipulation of Acc1 and Acc2 expression in the liver in rats with high-fat diet-induced hepatic steatosis and insulin resistance? The pharmacokinetic properties of antisense oligonucleotides (ASOs) used in the current study allowed us to examine in vivo reductions of target mRNA in the liver without causing any change in Acc expression in muscle. The results suggest that both Acc1 and Acc2 are involved in regulating fat oxidation in the liver, whereas only Acc1 synthe- sizes malonyl-CoA for fat synthesis, and that reducing both Acc1 and Acc2 expression with a single Acc ASO (Acc1 and -2) reverses hepatic steatosis and hepatic insulin resistance in high-fat-fed rats, providing a novel therapeutic option for the treatment of NAFLD.

\section{Results}

Acc ASOs decrease Acc expression and lower malonyl-CoA levels in rat livers. Three days after switching to a high-fat diet, an interval which allows for significant fat accumulation in the liver (6), rats were treated with ASO control (ASOctrl), Acc1, Acc2, or Acc1 and -2 ASOs at $25 \mathrm{mg} / \mathrm{kg}$ body weight twice a week for 4 weeks. Caloric intake and weight gain were similar in all treated groups and did not differ from those of high-fat-fed rats injected with saline. In comparison with ASOctrl, Acc1 and Acc2 ASO treatment reduced ACC1 (Figure 1A) and ACC2 mRNA (Figure 1B) levels, respectively, by approximately $80 \%$ in the liver. Whereas Acc1 and Acc2 ASOs reduced target expression in an Acc isoform-specific manner, treatment with the Acc1 and -2 ASO reduced both $A C C 1$ and $A C C 2$ mRNA by approximately $80 \%$ (Figure 1, $\mathrm{A}$ and $\mathrm{B})$. In contrast to the liver, no significant reductions were
A

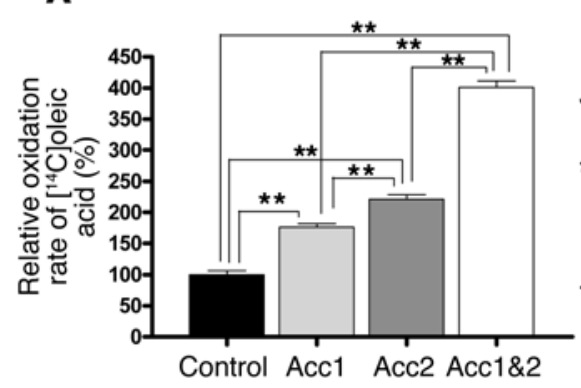

B

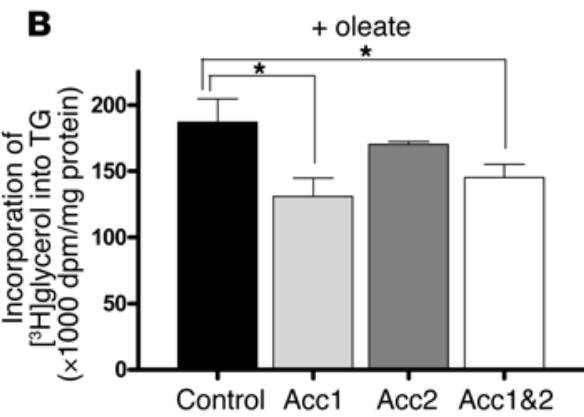

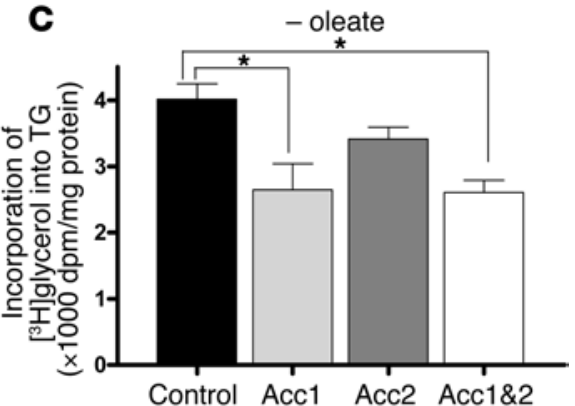

Figure 2

Effect of Acc ASOs on fatty acid oxidation and TG synthesis in primary rat hepatocytes. (A) Fat oxidation. (B and C) TG synthesis was assayed in the presence $(\mathbf{B})$ and absence $(\mathbf{C})$ of exogenous fatty acid (oleate). Data are expressed as mean values \pm SEM $(n=3)$. ${ }^{*} P<0.05 ;{ }^{* \star} P<0.01$. 

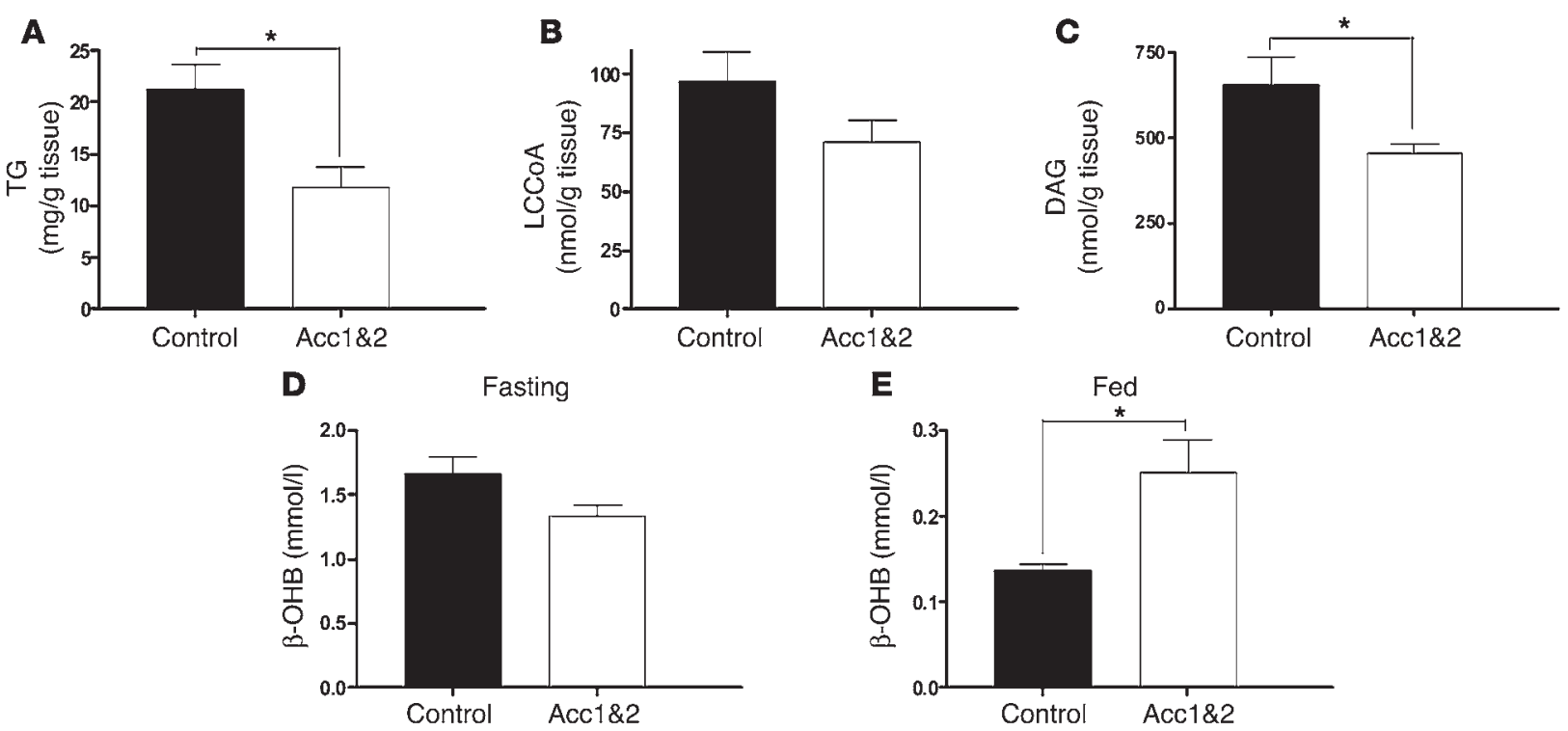

Figure 3

Acc1 and -2 ASO therapy lowers hepatic lipid levels in rats. TG (A), LCCoAs $(P=0.13)$, (B) and DAG (C) levels. Plasma ketones ( $\beta$-hydroxybutyrate $[\beta-\mathrm{OHB}])$ are similar in control and Acc1 and -2 ASO-treated rats in the fasting state (D) but are significantly increased in Acc1 and -2-treated rats in the fed state (E). Data are expressed as mean values \pm SEM of $4-6$ rats per treatment group. ${ }^{*} P<0.05$ versus ASOctrl treatment group.

found with any of the ASO-treated groups in muscle, consistent with previous findings (Figure 1, C and D) (15). Western blot analysis of liver protein extracts from rats injected with the Acc1, Acc2, and combined Acc1 and -2 ASOs confirmed isoform-selective knockdown of Acc protein expression and approximately 90\% knockdown of total Acc protein with the Acc1 and -2 ASO (Figure 1E). Western blotting also suggested that Acc1 is the dominant isoform, at least in terms of protein expression, in rat liver. Malonyl-CoA levels were not significantly altered by Acc1 or Acc2 ASO therapy, whereas the Acc1 and -2 ASO substantially lowered (49\%, $P<0.05)$ malonyl-CoA levels in the liver of refed rats (Figure $1 \mathrm{~F})$. Fasting malonyl-CoA levels, which are lower than those in refed rat livers, were similar in all groups (data not shown).

Impact of Acc ASOs on fatty acid oxidation and TG synthesis in primary rat hepatocytes. After overnight culture, primary rat hepatocytes transfected with ASOctrl, Acc1-, Acc2-, or Acc1 and -2 ASOs were incubated with medium containing $\left[{ }^{14} \mathrm{C}\right]$ oleic acid for another 2 hours for fatty acid oxidation measurement or $\left[{ }^{3} \mathrm{H}\right]$ glycerol for another 16 hours for TG synthesis measurement. Reduction of either Acc1 or Acc2 significantly increased fat oxidation, although the effect was significantly greater with the Acc2 ASO, which is believed to regulate malonyl-CoA levels in the immediate proximity of CPT1. This observation suggests that malonyl-CoA generated in the cytosol (via Acc1) also contributes to CPT1 regulation and is consistent with the fact that knockdown of

\section{Table 1} aminotransferase; nd, not determined. both isoforms synergistically increased fat oxidation (Figure 2A). In vitro fat synthesis was significantly inhibited only by the Acc1 and Acc1 and -2 ASOs (Figure 2, B and C), suggesting that Acc2 is solely involved in regulating fat oxidation whereas Acc1 contributes to the regulation of both fat synthesis and fat oxidation in hepatocytes. Given the in vitro evidence for a synergistic effect of combined ACC1 and ACC2 knockdown, further work focused on the in vivo impact of the Acc1 and - 2 ASO.

Acc1 and - 2 ASO reduces hepatic steatosis. Hepatic TGs were significantly lower in Acc1 and -2-treated animals than in controls (45\% reduction; $P<0.05)$ (Figure $3 \mathrm{~A}$ ). LCCoA also tended to be lower (26\% reduction; $P=0.13$ ), and diacylglycerol (DAG) levels were significantly $(30 \%$ reduction; $P<0.05)$ reduced in Acc1 and -2 ASOtreated animals (Figure 3, B and C). Plasma ketones are synthesized in the liver from acetyl-CoA derived from fatty acid oxidation and can, therefore, be used as an indirect marker of hepatic fatty acid oxidation. Interestingly, plasma ketones were only increased

Metabolic parameters during fasting, refed, and hyperinsulinemic-euglycemic clamp periods

\begin{tabular}{lcccccc} 
Group & \multicolumn{3}{c}{ Control $(\boldsymbol{n = 6})$} & \multicolumn{3}{c}{ Acc1 and $-\mathbf{2}(\boldsymbol{n}=\mathbf{9})$} \\
& Fasting & Refed & Clamp & Fasting & Refed & Clamp \\
Body weight $(\mathrm{g})$ & $410 \pm 9$ & $465 \pm 20$ & $417 \pm 18$ & $405 \pm 16$ & $457 \pm 15$ & $379 \pm 11$ \\
Glucose $(\mathrm{mg} / \mathrm{dl})$ & $118.8 \pm 2.6$ & $158.8 \pm 3.7$ & $101.8 \pm 1.4$ & $123.5 \pm 4.2$ & $169.8 \pm 9.3$ & $98.5 \pm 2.2$ \\
Insulin $(\mathrm{mU} / \mathrm{l})$ & $18 \pm 5$ & $79 \pm 6^{A}$ & $108 \pm 8$ & $21 \pm 7$ & $55 \pm 4$ & $106 \pm 8$ \\
FFA $(\mathrm{meq} / \mathrm{l})$ & $0.80 \pm 0.05$ & $0.49 \pm 0.11$ & $0.40 \pm 0.03$ & $0.83 \pm 0.05$ & $0.52 \pm 0.04$ & $0.39 \pm 0.06$ \\
Leptin $(\mathrm{ng} / \mathrm{ml})$ & $2.8 \pm 0.5$ & $10.6 \pm 1.2^{\mathrm{A}}$ & nd & $1.8 \pm 0.2$ & $4.8 \pm 1.0$ & nd \\
Adiponectin $(\mu \mathrm{g} / \mathrm{ml})$ & $1.9 \pm 0.2$ & nd & nd & $1.93 \pm 0.2$ & nd & nd \\
ALTB $(\mathrm{U} / \mathrm{l})^{27.9 \pm 1.7}$ & nd & nd & $24.4 \pm 2.2$ & nd & nd
\end{tabular}

Fasting refers to an overnight fast. Refed indicates animals that were fasted for 24 hours, then refed overnight. ${ }^{A} P<0.05$ control (refed) versus Acc 1 and -2 (refed). ${ }^{B}$ ALT in healthy chow fed rats was $<40 \mathrm{U} / \mathrm{l}$. ALT, alanine 

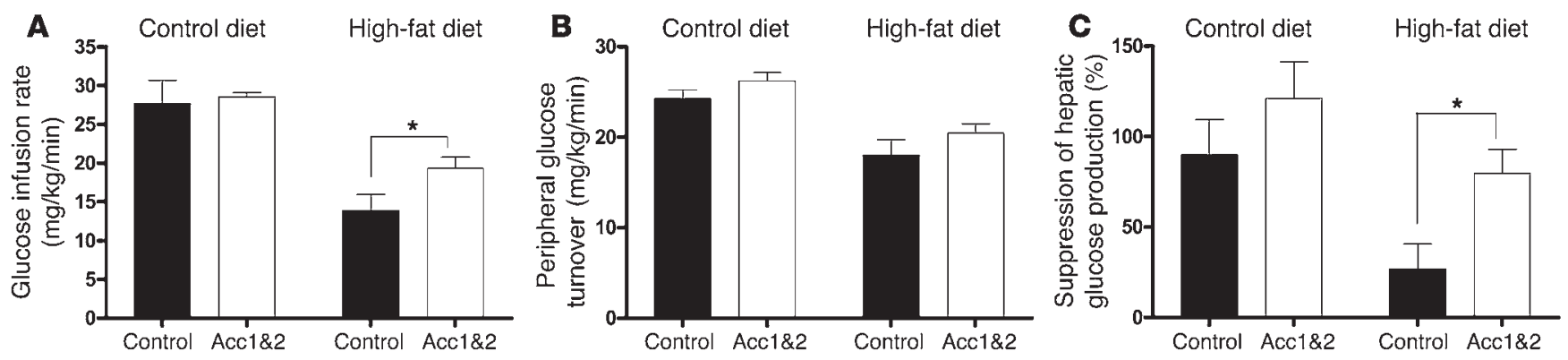

Figure 4

Acc1 and -2 ASO treatment significantly improved hepatic insulin sensitivity in high-fat-fed rats. Peripheral and hepatic insulin sensitivity were assessed by means of hyperinsulinemic-euglycemic clamps (A-C). (A) Glucose infusion rates; (B) peripheral glucose turnover; (C) suppression of HGP during hyperinsulinemic-euglycemic clamps. Data are expressed as mean values \pm SEM for $6-9$ rats per treatment group. ${ }^{\star} P<0.05$ versus ASOctrl treatment group.

in Acc1 and -2 ASO-treated animals in the fed state (83\% increase; $P<0.05$ ) (Figure 3, D and E), in keeping with the observed differences in malonyl-CoA concentrations (Figure 1F).

Acc 1 and - 2 ASO treatment improves hepatic insulin sensitivity. Caloric intake, total weight gain, and epididymal fat pad weight were similar in all treated groups (Table 1) and did not differ from those of high-fat-fed rats injected with saline (data not shown). Plasma alanine aminotransferase (ALT) levels were within the range seen in healthy chow-fed rats (Table 1), suggesting that at the doses used, inhibition of Acc does not cause hepatic toxicity. Fasting and refed plasma glucose and fatty acid levels were similar in both groups (Table 1), whereas refed plasma insulin and leptin levels were significantly reduced in Acc1 and -2 ASO-treated rats. The reduction in leptin levels during the fed state probably reflects the lower insulin levels (16). In order to independently assess hepatic and peripheral (predominantly muscle) insulin sensitivity, we performed hyperinsulinemic-euglycemic clamps (including radioisotope infusions) in ASO-treated rats. Glucose infusion rates were significantly higher in Acc1 and -2 ASO-treated high-fat-fed rats than in the ASOctrl group (Figure 4A). Whereas insulin-stimulated peripheral glucose metabolism was similar in both groups (Figure 4B), the ability of insulin to suppress endogenous glucose production was significantly increased by Acc1 and -2 ASO treatment (Figure 4C). Acc1 and -2 therapy did not alter peripheral or hepatic insulin sensitivity in chow-fed (control diet) rats (Figure 4, A-C). Neither the Acc1 nor the Acc2 ASO significantly altered insulin sensitivity (Supplemental Figure 1; supplemental material available online with this article; doi:10.1172/JCI27300DS1).

Effect of Acc1 and -2 ASO treatment on hepatic insulin signaling. We have previously shown that NAFLD is associated with activation of $\mathrm{PKC} \varepsilon$ as reflected by an increase in the plasma membrane/cytosol ratio of $\mathrm{PKC} \varepsilon$, an event which we believe may be causally related to the development of hepatic insulin resistance (6). Acc1 and -2 ASO therapy significantly reduced hepatic glucose production (HGP) during the hyperinsulinemic phase of the hyperinsulinemic-euglycemic clamp (Figure 5, A and B) and significantly reduced PKCE membrane translocation (Figure $5 \mathrm{C}$ ). These changes were associated with increased insulin-stimulated Akt2 activity (Figure 5D) and forkhead transcription factor FoxO1 (Foxo1) phosphorylation (Figure 5E). Foxo1 phosphorylation prevents Foxo1 from entering the nucleus and binding to promoters of target genes, such as phosphoenolpyruvate carboxykinase (PEPCK) and glucose- 6 phosphatase $(\mathrm{G} 6 \mathrm{P})(17,18)$. PEPCK and G6P are key regulators of
HGP, and insulin suppresses endogenous glucose production, at least in part, by lowering mRNA levels of PEPCK and G6P. While $P E P C K$ and G6P mRNA expression were similar in both groups in the fasted state, insulin-mediated suppression of their expression was significantly increased in Acc1 and -2 ASO-treated rats (Figure 5, F and $G$ ), explaining, at least in part, the reduction in HGP during the hyperinsulinemic phase of the clamp.

\section{Discussion}

It is becoming increasingly clear that hepatic lipid accumulation causes hepatic insulin resistance and contributes to the pathogenesis of type 2 diabetes (2). Acc catalyzes a key rate-controlling step in both de novo lipogenesis and fatty acid oxidation. Here we sought to evaluate the metabolic effects of isoform-specific knockdown of Acc1 and Acc2 in rat hepatocytes and the therapeutic potential of pharmacological inhibition of Acc expression in the liver in rats with diet-induced fatty liver. The results show that Acc1 and Acc2 ASOs selectively reduced ACC1 or ACC2 mRNA and protein expression in hepatocytes. The effects were both isoform specific and tissue selective, and there was no apparent compensatory increase in the nontargeted isoform, enabling us to directly assess the biological roles of each isoform in the liver for what we believe is the first time. Acc ASOs also reduced ACC expression in adipose tissue ( $~ 80 \%$, data not shown) but not in muscle. However, lowering Acc expression in adipose tissue did not alter epididymal fat-pad weight, body weight, plasma-free fatty acids, or 2-deoxyglucose uptake in adipose tissue (data not shown). While the tissue distribution patterns (Acc1 being predominantly expressed in lipogenic tissues and Acc2 in oxidative tissues) and intracellular localization (Acc2 has a mitochondrial targeting sequence) of each isoform previously led to the notion that cytosolic Acc1 regulates malonyl-CoA synthesis for incorporation into fatty acids, whereas Acc2 regulates mitochondrial fatty acid oxidation, this hypothesis has never been directly tested. Studies in ACC2-knockout mice suggested that malonyl-CoA in the liver was mainly produced by Acc1, which is the predominant isoform, but the fact that ACC2-null mice had reduced liver lipids suggested that malonyl-CoA produced by Acc2 in the immediate vicinity of CPT1 regulated fatty acid oxidation without producing a detectable change in malonyl-CoA levels (12). However, ACC2-null mice are substantially leaner than wild-type controls, making it difficult to discern the direct impact of ACC2 knockdown on lipids in the liver, and $A C C 1$ null mice are embryonic lethal (14). $A C C 1^{+/-}$mice are viable but appear to have normal Acc1 protein levels in hepatocytes 

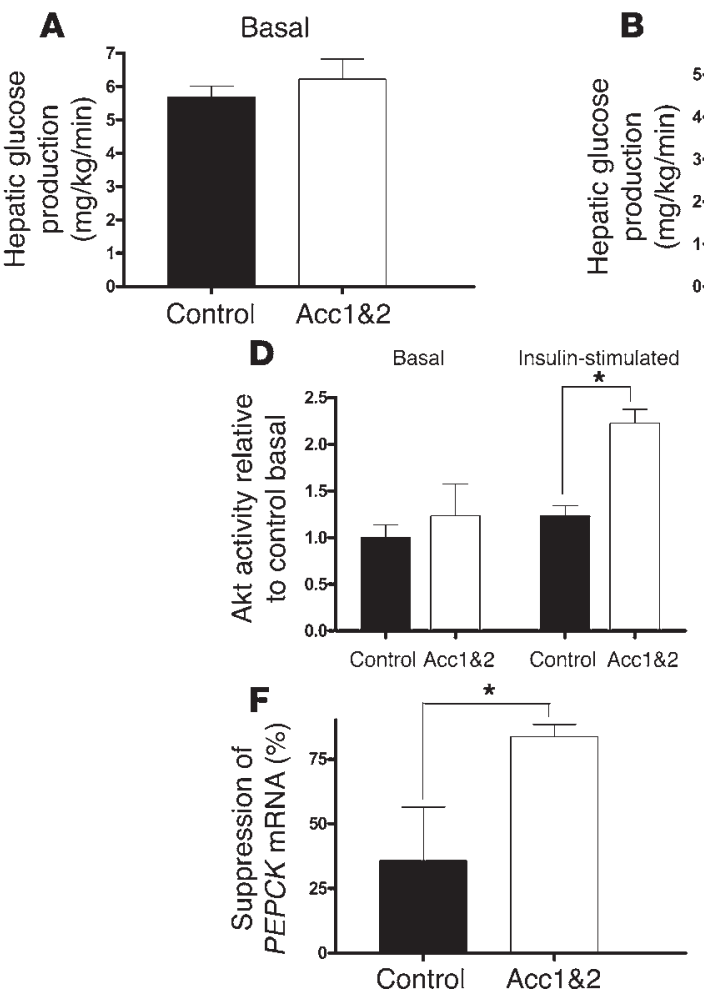

B
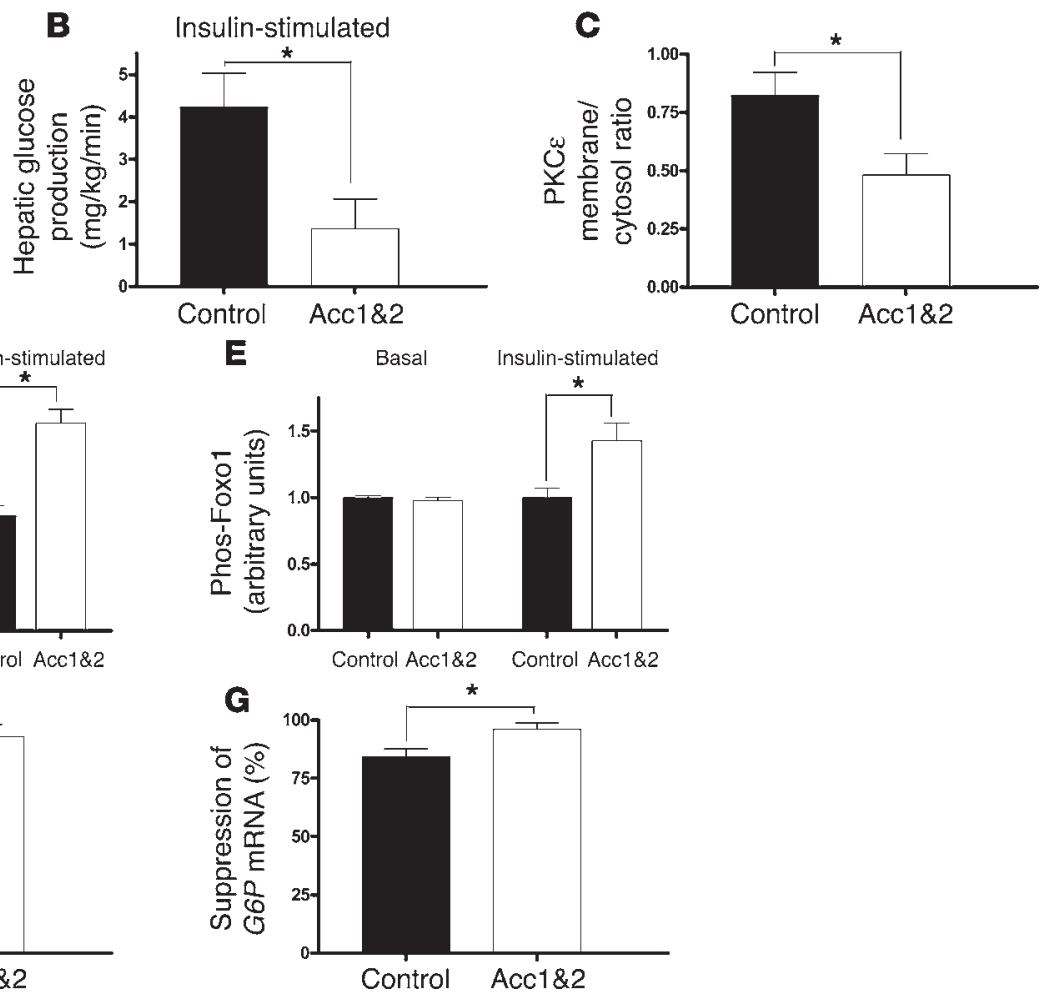

Figure 5

Acc1 and -2 ASO therapy improves hepatic insulin signaling. Acc1 and -2 ASO treatment does not alter basal HGP (A) but enhances insulin-mediated suppression of HGP in high-fat-fed rats (B). Reduced PKC $\varepsilon$ membrane translocation (C) may be directly involved in improving hepatic insulin signaling. This change is associated with increased Akt2 activity (D) and increased Foxo1 phosphorylation, which promotes nuclear exclusion of Foxo1, thereby lowering its transcriptional activity on the promoters of gluconeogenic genes, such as PEPCK and G6P. (D) Akt2 activity before (basal) and after 20 minutes insulin stimulation in ASOctrl and Acc1 and -2 ASO-treated rats. (E) Foxo1 phosphorylation was assessed before (basal) and after 20 minutes insulin stimulation by Western blotting using an antibody specific for serine256 phosphorylation. Data is expressed as the ratio of phosphorylated Foxo1 (Phos-Foxo1)/actin (loading control). (F) Suppression of hepatic PEPCK mRNA and (G) G6P mRNA expression during hyperinsulinemic-euglycemic clamps. ${ }^{*} P<0.05$ versus ASOctrl treatment group. Data are expressed as mean values \pm SEM for $4-6$ rats per treatment group. ${ }^{*} P<0.05$ versus ASOctrl treatment group.

(presumably as a result of altered translational regulation), making it difficult to assess the specific biological role of Acc 1 in this model (14). ASO-induced inhibition of Acc2 in rat hepatocytes significantly increased fat oxidation without altering malonyl-CoA levels in tissue extracts, in keeping with the notion that Acc2 may regulate malonyl-CoA levels in a localized pool in the proximity of CPT1. Reduction of Acc1 alone also significantly increased fat oxidation and inhibited fat synthesis without detectably altering hepatic malonyl-CoA levels. These data suggest that, at least in rat hepatocytes and in contrast to previous hypotheses (14), malonyl-CoA produced by Acc1 appears to regulate both fatty acid synthesis and fatty acid oxidation, whereas Acc2 is solely involved in regulating mitochondrial fat oxidation. This notion is further supported by the synergistic effect on fatty acid oxidation of simultaneously knocking down both Acc isoforms. Reduction of Acc 2 in addition to Acc1 has no further inhibitory effect on lipogenesis. The dominant role of Acc1 in regulating de novo lipogenesis is consistent with embryonic lethality in ACC1 knockouts as de novo synthesis of long-chain fatty acids is required during mouse embryo development.

High-fat feeding in rats causes hepatic lipid accumulation and insulin resistance within 3 days (6). The pattern of LCCoA accumulation (linoleic acid [18:2] is the major fatty acid constituent of the diet used in this study and the major detectable LCCoA in liver extracts in these rats) suggests that this is primarily a consequence of fatty acid re-esterification rather than de novo lipogenesis (6). Fatty acyl-CoA 18:2 is also the dominant LCCoA and the dominant fatty acid in the DAG fraction in rat liver after 4 weeks of high-fat feeding (data not shown), suggesting that re-esterification remains the dominant factor in the development of steatosis in high-fat-fed rats. Thus, we believe that the in vivo effects of the Acc1 and - 2 ASO are predominantly a result of increased fat oxidation rather than a tendency to inhibit fat synthesis in this model. Indirect support for this notion is provided by the increase in plasma ketones, a marker of hepatic fat oxidation. Interestingly, the rise in plasma ketones was only detectable in the fed state. Malonyl-CoA levels are lower in the fasting state in rat liver than in the fed state, a result of hormonal and allosteric regulation of Acc expression and activity (9), and it is not therefore particularly surprising that reduction of Acc1 and Acc2 expression had no further effect on fasting ketone levels. Although fat oxidation occurs to a significantly greater extent in the fasting state than in the fed state, we believe that with time a relatively modest increase in fat oxidation in the fed state results in lower liver lipid levels and improved hepatic insulin sensitivity. In contrast to the dramatic changes in 
body weight seen in ACC2-null mice (12), Acc inhibition in the liver in rats does not lower body weight. As the liver of a 400-g SpragueDawley rat only weighs about $15 \mathrm{~g}$ and the rats had unrestricted access to high-fat food, it is not particularly surprising that selectively increasing fat oxidation in the liver in the fed state did not result in a significant change in body weight. It would likely take longer to see significant weight reduction with liver-specific Acc reduction, as opposed to a global loss of Acc activity observed in the ACC2-null mice. Recent data suggest that lowering malonylCoA levels in the hypothalamus could increase food intake (19), an important concern for those developing pharmacological Acc inhibitors; however, the intraperitoneally administered ASOs used in this study do not alter target gene expression in the hypothalamus (15), and we did not detect any differences in food intake.

A growing body of evidence implicates serine phosphorylation of insulin signaling intermediates in the pathogenesis of insulin resistance $(20,21)$. Several candidate serine/threonine kinases exist, one of which is the novel PKC (nPKC) family. We have previously suggested that hepatic lipid accumulation in high-fat-fed rodents is associated with PKCe translocation to the plasma membrane (6), a finding which was reversed by the Acc1 and -2 ASO. While TGs are typically associated with both hepatic and muscle insulin resistance, they are widely perceived to be relatively inert biologically. Instead, lipid intermediates such as LCCoAs and DAGs are more favored candidates for triggering fat-induced insulin resistance. The fact that mice deficient in mitochondrial glycerol phosphate acyltransferase (GPAT) are more insulin sensitive than wild-type mice fed a high-fat diet despite substantial increases in hepatic LCCoAs suggests that DAG is a better candidate than LCCoA in this process (22). DAG is also a known activator of PKC (23-25), making it an excellent candidate for this role. Acc1 and -2 ASO therapy decreased TG, LCCoA, and DAG accumulation, reduced PKCE membrane translocation, and improved insulin-stimulated Akt2 activity. In addition, this increased Foxo 1 phosphorylation and improved insulin-stimulated suppression of gluconeogenic gene expression.

Lipid accumulation in liver and skeletal muscle is a feature of both obesity and lipodystrophy and has been strongly implicated in the pathogenesis of insulin resistance and type 2 diabetes $(21,26)$. A number of strategies have been employed in efforts to limit ectopic lipid accumulation, perhaps the most effective being to limit food intake either by simple dieting (7) or by restricting gastric capacity (gastric banding) (27). Leptin replacement in leptin-deficient lipodystrophic mice (28) and humans $(29,30)$ has a similar effect, with dramatic metabolic benefits. However, given the limited impact of these approaches in most people with fatty liver, several attempts have been made to increase fat oxidation pharmacologically. A number of Acc small molecule inhibitors (all of which appear to inhibit both Acc1 and Acc2) have been tested in preclinical studies (31). In general, they do increase fat oxidation and improve metabolic indices. However, Acc inhibition in both the hypothalamus, where lowering malonyl-CoA levels may increase food intake, and pancreas, where lowering malonyl-CoA levels appears to reduce insulin secretion at the cost of higher glucose levels in obese mice (31), may be disadvantageous. Alternative strategies have included adenoviral delivery of excess uncoupling protein 1 (UCP1) (32) or malonyl-CoA decarboxylase (MCD) (33) to the liver. Both approaches led to significant reductions in liver lipids and improvements in hepatic insulin sensitivity.
In summary, the current study suggests that both Acc1 and Acc2 are involved in regulating fat oxidation in hepatocytes while Acc1, the dominant isoform in rat liver, is the sole regulator of fatty acid synthesis. Furthermore, in this model, combined reduction of both isoforms is required to significantly lower hepatic malonylCoA levels, increase fat oxidation in the fed state, reduce lipid accumulation, and improve insulin action in vivo. Thus, hepatic Acc1 and -2 inhibitors may be useful in the treatment of NAFLD and hepatic insulin resistance.

\section{Methods}

Animals. All rats were maintained in accordance with the institutional Animal Use and Care Committees of Yale University School of Medicine. Healthy male Sprague-Dawley rats weighing 200-225 g were obtained from Charles River Laboratories and acclimated for 1 week after arrival before initiation of the experiment. Rats received food and water ad libitum and were maintained on a 12/12-hour light/dark cycle (lights on at 6:30 am). They were housed individually, and food consumption and body weight were monitored. Rats received either regular rodent chow (60\% carbohydrate $/ 10 \%$ fat $/ 30 \%$ protein calories) or a high-fat diet ( $26 \%$ carbohydrate $/ 59 \%$ fat $/ 15 \%$ protein calories). Safflower oil was the major constituent of the high-fat diet (Dyets Inc.). We have previously shown that this diet produces hepatic steatosis and hepatic insulin resistance within 3 days (6). ASO therapy was initiated i.p. 3 days after commencing the high-fat diet. All ASOs (control, Acc1, Acc2, and Acc1 and -2) were prepared in normal saline, and the solutions were sterilized through a $0.2-\mu \mathrm{m}$ filter. Rats were dosed with ASO solutions or saline twice per week via i.p. injection at a dose of $50 \mathrm{mg} / \mathrm{kg} / \mathrm{wk}$ for 4 weeks. During the treatment period, body weight and food intake were measured twice weekly. The Yale Animal Care and Use Committee approved all protocols.

Selection of rat ACC ASOs. Rapid throughput screens were performed in vitro to identify rat $A C C 1$ - and $A C C 2$-specific ASOs. ASOs were screened in rat primary hepatocytes for their ability to inhibit ACC 1 or ACC 2 mRNA expression, as described previously (34). The most potent ASOs were further characterized, and the lead ASOs were selected for in vivo studies. An in vivo approach was used to screen for the Acc1 and -2 ASO inhibitor. In brief, ASOs were designed to target regions of nucleotide identity between ACC1 (GenBank accession number XM_109883) and ACC2 (GenBank accession number XM_ 132282 ) and were administered to Sprague-Dawley rats at $75 \mathrm{mg} / \mathrm{kg} / \mathrm{wk}$ for 2 weeks. Target reduction in the liver revealed approximately $80 \%$ reduction of both ACC1 and ACC2 mRNA with the lead ASO. The final lead rat Acc1, Acc2, and Acc1 and -2 ASOs used in the study were as follows: ISIS-338292, 5'-CGTGGGATGCCTTCTGCTCT-3' (position 5116-5136bp NM_022193); ISIS-189594, 5'-GAGTTCCTCTGCTGACTGGC-3' (241-261bp AB004329); and ISIS-362037, 5'-CCTCATCTGGGTTTTCGCTG-3' (1530-1550bp NM_ 022193 and 1950-1970bp AB004329, respectively). All ASOs were synthesized as 20-base phosphorothioate chimeric ASOs, where bases 1-5 and 16-20 were modified with 2'-O-(2-methoxy)-ethyl (2'-MOE). This chimeric design has been shown to provide both increased nuclease resistance and mRNA affinity while maintaining the robust RNase $\mathrm{H}$ terminating mechanism utilized by these types of ASOs (35). These benefits result in an attractive in vivo pharmacological and toxicological profile for 2'-MOE chimeric ASOs. The control ASO, ISIS-141923, has the same design and chemistry as the Acc ASOs with the following sequence: $5^{\prime}$-CCTTCCCTGAAGGTTCCTCC-3'. It does not have perfect complementarity to any known gene in public databases.

Determination of fatty acid oxidation and TG synthesis in transfected rat hepatocytes in vitro. Primary rat hepatocytes were isolated as previously described and plated onto collagen-coated $25-\mathrm{cm}^{2}$ flasks for fatty acid oxidation measurement or 60-mm plates for TG synthesis measurement (36). Hepatocytes were treated with ASO $(150 \mathrm{nM})$ and Lipofectin (Invitrogen Corp.) mixture for 4 hours in serum-free William's E media (Invitrogen Corp.). ASO and Lipo- 
fectin were mixed in a ratio of $3 \mu \mathrm{g}$ of Lipofectin for every $1 \mathrm{ml}$ of $100 \mathrm{nM}$ ASO concentration. After 4 hours, ASO reaction mixture was replaced with normal maintenance media (William's E media with $10 \%$ FBS and $10 \mathrm{nM}$ insulin). The cells were incubated under normal conditions for 20-24 hours, and then fatty acid $\left(\left[{ }^{14} \mathrm{C}\right]\right.$ oleate) oxidation and TG synthesis (incorporation of $\left[{ }^{3} \mathrm{H}\right]$ glycerol into TGs) were measured as described previously (36).

Hyperinsulinemic-englycemic clamp studies. Seven days prior to the hyperinsulinemic-euglycemic clamp studies, indwelling catheters were placed into the right internal jugular vein extending to the right atrium and the left carotid artery extending to the aortic arch. After an overnight fast, $\left[3-{ }^{3} \mathrm{H}\right]$-glucose (HPLC purified; PerkinElmer) was infused at a rate of $0.33 \mu \mathrm{Ci} / \mathrm{min}$ for 2 hours to assess the basal glucose turnover. Following the basal period, the hyperinsulinemic euglycemic clamp was conducted for 120 minutes with a primed/continuous infusion of human insulin $(200 \mathrm{mU} / \mathrm{kg}$ prime, $4 \mathrm{mU} / \mathrm{kg} / \mathrm{min}$ infusion) (Novo Nordisk) and a variable infusion of $20 \%$ dextrose to maintain euglycemia $(\sim 100 \mathrm{mg} / \mathrm{dl})$. $\left[3-{ }^{3} \mathrm{H}\right]$-glucose was infused at a rate of $1.0 \mu \mathrm{Ci} / \mathrm{min}$ throughout the clamps. A25- $\mu$ Cibolus of 2-deoxy-D-[1-14 $\mathrm{C}]$ glucose (PerkinElmer) was injected 75 minutes into the clamp to estimate the rate of insulin-stimulated tissue glucose uptake. At the end of the clamp, rats were anesthetized with pentobarbital sodium injection $(150 \mathrm{mg} / \mathrm{kg})$ and all tissues were taken within 4 minutes, frozen immediately using liquid $\mathrm{N}_{2}$-cooled aluminum tongs, and stored at $-80^{\circ} \mathrm{C}$ for subsequent analysis.

Biochemical analysis and calculations. Plasma glucose was analyzed during the clamps using $10 \mu \mathrm{l}$ plasma by a glucose oxidase method on a Beckman Coulter glucose analyzer II (Beckman Coulter). Plasma insulin, adiponectin, and leptin were measured by radioimmunoassay, using kits from Linco Research Inc. The adiponectin kit was primarily designed to measure mouse adiponectin, but showed significant cross-reactivity with rat adiponectin. Plasma fatty acid concentrations were determined using an acylCoA oxidase-based colorimetric kit (Wako Pure Chemical Industries Ltd.). For the determination of plasma ${ }^{3} \mathrm{H}$-glucose, plasma was deproteinized with $\mathrm{ZnSO}_{4}$ and $\mathrm{Ba}(\mathrm{OH})_{2}$, dried to remove ${ }^{3} \mathrm{H}_{2} \mathrm{O}$, resuspended in water, and counted in scintillation fluid (Ultima Gold; PerkinElmer) on a Beckman scintillation counter. Rates of basal and insulin-stimulated wholebody glucose turnover were determined as the ratio of the $\left[3-{ }^{3} \mathrm{H}\right]$-glucose infusion rate $(\mathrm{dpm})$ to the specific activity of plasma glucose $(\mathrm{dpm} / \mathrm{mg})$ at the end of the basal period and during the final 30 minutes of the clamp experiment, respectively. HGP was determined by subtracting the glucose infusion rate from the rate of total glucose appearance.

Tissue lipid measurement. The solid-phase extraction and purification of medium, long-chain, and very long-chain fatty acyl-CoAs from liver have been described previously $(37,38)$. After purification, fatty acyl-CoA fractions were dissolved in methanol/ $\mathrm{H}_{2} \mathrm{O}(1: 1, \mathrm{v} / \mathrm{v})$ and subjected to liquid chromatography/mass spectrometry/mass spectrometry (LC/MS/MS) analysis. A turbo ion spray source was interfaced with an API 3000 tandem mass spectrometer (Applied Biosystems) in conjunction with 2 PerkinElmer 200 Series micro pumps and a 200 Series autosampler (PerkinElmer). The DAG extraction and analysis were performed as previously described (25, 39). Total DAG content is expressed as the sum of individual species. Tissue TG was extracted using the method of Bligh and Dyer (37) and measured using a DCL TG Reagent (Diagnostic Chemicals Ltd).

Tissue malonyl-CoA content. Approximately $100 \mathrm{mg}$ of liver tissue was homogenized in ice-cold $10 \%$ trichloroacetic acid with the addition of ${ }^{13} \mathrm{C}_{3}$-malonyl-CoA internal standard. After centrifugation, the supernatant was applied to a preconditioned cartridge (C18 Waters Sep-Pak Cartridge, Waters Corp.) and, following washing steps, eluted with $65 \%$ ethanol containing $0.1 \mathrm{M} \mathrm{NH}_{4}$-acetate and 50\% methanol. Samples were lyophilized and redissolved in water. Malonyl-CoA and acetyl-CoA were separated by HPLC with isocratic $15 \%$ methanol and $10 \mathrm{mM}$ ammonium acetate. Malonyl-CoA was measured using LC/MS/MS analysis.
Liver Akt2 activity, Foxo1 phosphorylation, and PKCE membrane translocation. Akt2 activity and Foxo1 phosphorylation were assessed in protein extracts from livers harvested after short-term $(20 \mathrm{~min})$ insulin stimulation (200 mU/kg prime, $4 \mathrm{mU} / \mathrm{kg} / \mathrm{min}$ infusion) in combination with $20 \% \mathrm{dex}$ trose to maintain euglycemia $(100 \mathrm{mg} / \mathrm{dl})$. Assays were performed according to methods previously described $(6,40,41)$. Primary antibodies used for these experiments were rabbit polyclonal IgGs (Cell Signaling Technology). For PKC $\varepsilon$ membrane translocation, $50 \mu \mathrm{g}$ of crude membrane and cytosol protein extracts were resolved by SDS-PAGE using $8 \%$ gel and electroblotted onto polyvinylidene difluoride membrane (DuPont) using a semidrytransfer cell (Bio-Rad). The membrane was then blocked for 2 hours at room temperature in PBS-Tween (PBS-T) $\left(10 \mathrm{mmol} / \mathrm{liter} \mathrm{NaH}_{2} \mathrm{PO} 4\right.$, $80 \mathrm{mmol} /$ liter $\mathrm{Na}_{2} \mathrm{HPO}_{4}, 0.145 \mathrm{~mol} /$ liter NaCl, and 0.1\% Tween-20, pH 7.4) containing $5 \%(\mathrm{w} / \mathrm{v})$ nonfat dried milk, washed twice, and then incubated overnight with rabbit anti-peptide antibody against PKCe (Santa Cruz Biotechnology Inc.) diluted 1:100 in rinsing solution. After further washings, membranes were incubated with horseradish peroxidase-conjugated IgG fraction of goat anti-rabbit IgG (Bio-Rad) diluted 1:5,000 in PBS-T for 2 hours. PKC $\varepsilon$ translocation was expressed as the ratio of membrane bands to cytosol bands (arbitrary units).

Quantitative RT-PCR-based gene expression analysis. Quantitative RT-PCR was performed essentially as previously described (42). Briefly, RNA was isolated using a commercially available kit (QIAGEN RNeasy Kit; QIAGEN) in combination with DNase digest treatment. After $1.0 \mu \mathrm{g}$ of total RNA was reverse transcribed (Stratagene) with an oligo-prime, PCR was performed with a DNA Engine Opticon 2 System (MJ Research; Bio-Rad) using SYBR green qPCR dye kit (Stratagene). Primer sequences are presented in Supplemental Table 1. After the PCR, standard curves were constructed from the standard reactions for each target mRNA species and $18 \mathrm{~s}$ mRNA by plotting values for $\mathrm{Ct}$ (the number of PCR cycles at which the fluorescence signal exceeds background) versus log cDNA input (in nanograms, arbitrarily assigned). The $\mathrm{Ct}$ readings for each of the samples were then used to calculate the amount of mRNA for each target gene relative to the standard. For each sample, results were normalized by dividing the amount of target gene mRNA by the amount of $18 \mathrm{~s}$ mRNA.

Statistics. All data are expressed as mean \pm SEM. Two-tailed Student's $t$ tests or 1-way ANOVA plus Tukey HSD (honestly significant difference) multiple comparisons were performed on data with significance set at a $P$ value of 0.05 .

\section{Acknowledgments}

We thank Jiaying Dong and Anthony J. Romanelli for expert technical assistance with the studies. We also thank Aida Groszmann for performing the hormone assays. This work was supported by grants from the US Public Health Service (R01 DK-40936 and P30 DK-45735 to G.I. Shulman) and a Distinguished Clinical Scientist Award from the American Diabetes Association. G.I. Shulman is an investigator of the Howard Hughes Medical Institute. D.B. Savage is supported by the Wellcome Trust.

Received for publication November 2, 2005, and accepted in revised form December 13, 2005.

Address correspondence to: Gerald I. Shulman, The Anlyan Centre S269, PO Box 9012, 300 Cedar Street, Yale University School of Medicine, New Haven, Connecticut 06510, USA. Phone: (203) 785-5447; Fax: (203) 737-4059; E-mail: gerald.shulman@yale.edu.

David B. Savage and Cheol Soo Choi contributed equally to this work. 
1. Browning, J.D., et al. 2004. Prevalence of hepatic steatosis in an urban population in the United States: impact of ethnicity. Hepatology. 40:1387-1395.

2. Browning, J.D., and Horton, J.D. 2004. Molecular mediators of hepatic steatosis and liver injury. J. Clin. Invest. 114:147-152. doi:10.1172/ JCI200422422.

3. Kim, H.J., et al. 2004. Metabolic significance of nonalcoholic fatty liver disease in nonobese, nondiabetic adults. Arch. Intern. Med. 164:2169-2175.

4. Seppala-Lindroos, A., et al. 2002. Fat accumulation in the liver is associated with defects in insulin suppression of glucose production and serum free fatty acids independent of obesity in normal men. J. Clin. Endocrinol. Metab. 87:3023-3028.

5. Kim, J.K., et al. 2001. Tissue-specific overexpression of lipoprotein lipase causes tissue-specific insulin resistance. Proc. Natl. Acad. Sci. U. S. A. 98:7522-7527.

6. Samuel, V.T., et al. 2004. Mechanism of hepatic insulin resistance in non-alcoholic fatty liver disease. J. Biol. Chem. 279:32345-32353.

7. Petersen, K.F., et al. 2005. Reversal of nonalcoholic hepatic steatosis, hepatic insulin resistance, and hyperglycemia by moderate weight reduction in patients with type 2 diabetes. Diabetes. 54:603-608.

8. Munday, M.R., and Hemingway, C.J. 1999. The regulation of acetyl-CoA carboxylase-a potential target for the action of hypolipidemic agents. Adv. Enzyme Regul. 39:205-234.

9. Munday, M.R. 2002. Regulation of mammalian acetyl-CoA carboxylase. Biochem. Soc. Trans. 30:1059-1064.

10. Wakil, S.J., Stoops, J.K., and Joshi, V.C. 1983. Fatty acid synthesis and its regulation. Annu. Rev. Biochem. 52:537-579.

11. McGarry, J.D., and Brown, N.F. 1997. The mitochondrial carnitine palmitoyltransferase system. From concept to molecular analysis. Eur. J. Biochem. 244:1-14.

12. Abu-Elheiga, L., Matzuk, M.M., Abo-Hashema, K.A., and Wakil, S.J. 2001. Continuous fatty acid oxidation and reduced fat storage in mice lacking acetyl-CoA carboxylase 2. Science. 291:2613-2616.

13. Abu-Elheiga, L., et al. 2000. The subcellular localization of acetyl-CoA carboxylase 2. Proc. Natl. Acad. Sci.U. S. A. 97:1444-1449.

14. Abu-Elheiga, L., et al. 2005. Mutant mice lacking acetyl-CoA carboxylase 1 are embryonically lethal. Proc. Natl. Acad. Sci. U. S. A. 102:12011-12016.

15. Buettner, C., et al. 2005. Severe impairment in liver insulin signaling fails to alter hepatic insulin action in conscious mice. J. Clin. Invest. 115:1306-1313. doi:10.1172/JCI200523109.

16. Saladin, R., et al. 1995. Transient increase in obese gene expression after food intake or insulin administration. Nature. 377:527-529.

17. Accili, D., and Arden, K.C. 2004. FoxOs at the crossroads of cellular metabolism, differentiation, and transformation. Cell. 117:421-426.

18. Frescas, D., Valenti, L., and Accili, D. 2005. Nuclear trapping of the forkhead transcription factor FoxO1 via Sirt-dependent deacetylation promotes expression of glucogenetic genes. J. Biol. Chem. 280:20589-20595.

19. Hu, Z., Cha, S.H., Chohnan, S., and Lane, M.D. 2003. Hypothalamic malonyl-CoA as a mediator of feeding behavior. Proc. Natl. Acad. Sci. U. S. A. 100:12624-12629.

20. Savage, D.B., Petersen, K.F., and Shulman, G.I 2005. Mechanisms of insulin resistance in humans and possible links with inflammation. Hypertension. 45:828-833.

21. Shulman, G.I. 2000. Cellular mechanisms of insulin resistance. J. Clin. Invest. 106:171-176.

22. Neschen, S., et al. 2005. Prevention of hepatic steatosis and hepatic insulin resistance in mitochondrial acyl-CoA: glycerol-sn-3-phosphate acyltransferase 1 knockout mice. Cell Metab. 2:55-65.

23. Shmueli, E., Alberti, K.G., and Record, C.O. 1993. Diacylglycerol/protein kinase C signalling: a mechanism for insulin resistance? J. Intern. Med. 234:397-400.

24. Considine, R.V., et al. 1995. Protein kinase C is increased in the liver of humans and rats with non-insulin-dependent diabetes mellitus: an alteration not due to hyperglycemia. J. Clin. Invest. 95:2938-2944

25. Yu, C., et al. 2002. Mechanism by which fatty acids inhibit insulin activation of insulin receptor substrate-1 (IRS-1)-associated phosphatidylinositol 3-kinase activity in muscle. J. Biol. Chem. 277:50230-50236.

26. Friedman, J. 2002. Fat in all the wrong places. Nature. 415:268-269.

27. Sjostrom, L., et al. 2004. Lifestyle, diabetes, and cardiovascular risk factors 10 years after bariatric surgery. N. Engl. J. Med. 351:2683-2693.

28. Shimomura, I., Hammer, R.E., Ikemoto, S., Brown, M.S., and Goldstein, J.L. 1999. Leptin reverses insulin resistance and diabetes mellitus in mice with congenital lipodystrophy. Nature. 401:73-76.

29. Petersen, K.F., et al. 2002. Leptin reverses insulin resistance and hepatic steatosis in patients with severe lipodystrophy. J. Clin. Invest. 109:1345-1350. doi:10.1172/JCI200215001.

30. Oral, E.A., et al. 2002. Leptin-replacement therapy for lipodystrophy. N. Engl. J. Med. 346:570-578.

31. Harwood, H.J., Jr. 2005. Treating the metabolic syndrome: acetyl-CoA carboxylase inhibition. Expert Opin. Ther. Targets. 9:267-281.

32. Ishigaki, Y., et al. 2005. Dissipating excess energy stored in the liver is a potential treatment strategy for diabetes associated with obesity. Diabetes. 54:322-332.

33. An, J., et al. 2004. Hepatic expression of malonylCoA decarboxylase reverses muscle, liver and wholeanimal insulin resistance. Nat. Med. 10:268-274.

34. Watts, L.M., et al. 2005. Reduction of hepatic and adipose tissue glucocorticoid receptor expression with antisense oligonucleotides improves hyperglycemia and hyperlipidemia in diabetic rodents without causing systemic glucocorticoid antagonism. Diabetes. 54:1846-1853.

35. McKay, R.A., et al. 1999. Characterization of a potent and specific class of antisense oligonucleotide inhibitor of human protein kinase C-alpha expression. J. Biol. Chem. 274:1715-1722.

36. Yu, X.X., et al. 2005. Antisense oligonucleotide reduction of DGAT2 expression improves hepatic steatosis and hyperlipidemia in obese mice. Hepatology. 42:362-371.

37. Bligh, E.G., and Dyer, W.J. 1959. A rapid method of total lipid extraction and purification. Can. J. Biochem. Physiol. 37:911-917.

38. Neschen, S., et al. 2002. Contrasting effects of fish oil and safflower oil on hepatic peroxisomal and tissue lipid content. Am. J. Physiol. Endocrinol. Metab. 282:E395-E401.

39. Neschen, S., et al. 2005. Prevention of hepatic steatosis and hepatic insulin resistance in mitochondrial acyl-CoA: glycerol-sn-3-phosphate acyltransferase 1 knockout mice. Cell Metab. 2:55-65

40. Alessi, D.R., et al. 1996. Mechanism of activation of protein kinase B by insulin and IGF-1. EMBO J. 15:6541-6551.

41. Qu, X., Seale, J.P., and Donnelly, R. 1999. Tissue and isoform-selective activation of protein kinase $\mathrm{C}$ in insulin-resistant obese Zucker rats - effects of feeding. J. Endocrinol. 162:207-214.

42. Savage, D.B., et al. 2001. Resistin/Fizz3 expression in relation to obesity and peroxisome proliferator-activated receptor-gamma action in humans. Diabetes. 50:2199-2202. 